
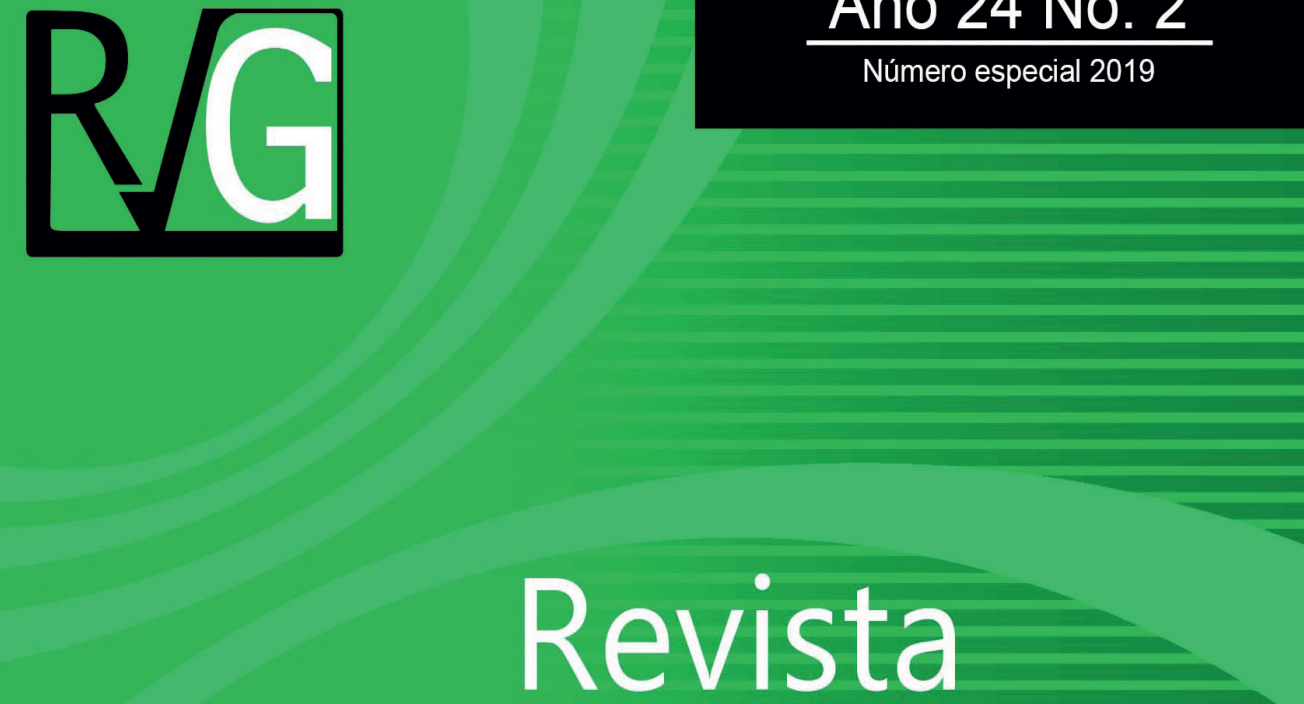

Venezolana de

$\frac{1}{6}$

$\frac{\mathbb{O}}{\frac{1}{0}}$
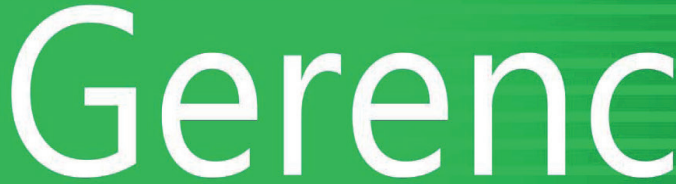

0

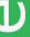

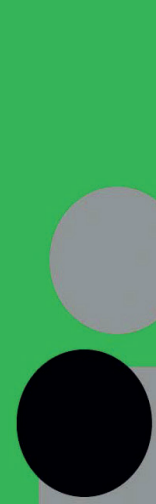

ro

u

$>$

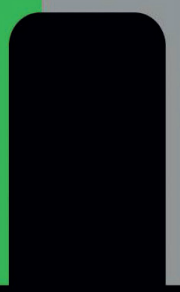




\title{
Innovación Empresarial: Factor de competitividad y calidad de vida en Popayán, Colombia
}

\author{
Saldarriaga Salazar, María Eugenia ${ }^{1}$ \\ Guzmán González, María Fernanda ${ }^{2}$ \\ Concha Cerón, Eduardo Andrés ${ }^{3}$
}

\section{Resumen}

En las actuales condiciones competitivas, las micro, pequeñas y medianas empresas de Popayán (Colombia), deben mantener su desarrollo económico en mercados competidos. El objetivo es determinar el rol de la innovación en empresas de Popayán, como factor de competitividad para lograr mejoramiento de la calidad de vida en la región. La investigación es cualitativa, exploratoria y utiliza como instrumento la entrevista semiestructura en treinta empresas. Los resultados indican que la innovación

Recibido: 11-09-19 Aceptado: 24-10-19

$1 \quad$ Magister en Dirección de Marketing (Universidad de Viña del Mar, Chile), Administradora de Empresas, Docente de Institución Universitaria Colegio Mayor del Cauca, Popayán, Colombia, Directora del grupo de investigación Historeo, Investigadora Asociada (Colciencias). E-mail: msaldarriaga@unimayor.edu.co. Código Orcid: orcid.org/0000-0002-3608-5988.

2 Magister en Educación: Desarrollo Humano (Uni Cauca, Colombia), Licenciada en Leguas Modernas: inglés - francés, Catedrática, Institución Universitaria Colegio Mayor del Cauca. Coinvestigadora del grupo de investigación Historeo. Popayán, Colombia. E-mail: mfdaguzman@gmail.com. Código Orcid: orcid.org/0000-0001-6144-1465

3 MBA - Magister en Administración de Empresas, (Universidad Javeriana, Colombia), Administrador de Empresas, Asesor Empresarial, Investigador Externo del grupo de investigación Historeo, Institución Universitaria Colegio Mayor del Cauca, Popayán, Colombia, E-mail: ea_concha@hotmail.com. Código Orcid: orcid.org/0000-0003-1212-2147 
se fundamenta en lineamientos o tecnologías para el mejoramiento continuo en procesos, productos y servicios que se aplican como ventaja competitiva; enmarcados en factores externos para estar al tanto de los cambios del entorno y aprovechamiento de mejoras tecnológicas, y factores internos en implementación de dirección abierta, participativa, descentralización en toma de decisiones, motivación del personal y compromiso por innovación. Como conclusiones, se obtiene que, la innovación es una ventaja competitiva que permite la diferenciación y adaptarse a cambios del medio. Se puede observar, que las empresas ofrecen productos y servicios mejorados, y para innovar, no invierten grandes cantidades de recursos, sin embargo, han logrado obtener innovación y, por lo tanto, mejoramiento en la calidad de vida.

Palabras clave: innovación empresarial; competitividad; calidad de vida

\title{
Business Innovation: Competitiveness Factor and Quality of Life in Popayán, Colombia
}

\begin{abstract}
Under today's competitive conditions, micro, small and medium-sized enterprises in Popayán (Colombia) must maintain their economic development in competitive markets. The objective is to determine the role of innovation in Popayán enterprises, as a competitiveness factor to achieve improvement of the quality of life in the region. The research is qualitative, exploratory and uses as an instrument the semistructure interview in thirty companies. The main results indicate that innovation is based on guidelines or technologies for continuous improvement in processes, products and services that are applied as a competitive advantage; framed in external factors to be aware of changes in the environment and take advantage of technological improvements, and internal factors in implementation of open, participatory management, decentralization in decisionmaking, motivation of staff and commitment to innovation. As important conclusions, it is obtained that, innovation is a competitive advantage that allows the differentiation, and adapt to changes of the environment. It can be seen that companies offer improved products and services, and to innovate, they do not invest large amounts of resources, however, they have managed to achieve innovation and, therefore, improvement in quality of life.
\end{abstract}

Keywords: business Innovation; competitiveness; quality of life. 


\section{Introducción}

Hoy la innovación ha tomado gran importancia en el sector empresarial, su aplicación se considera fundamental cuando se requiere lograr el éxito empresarial en un medio competido para ser sostenibles en el mercado, así mismo, desde el punto de vista empresarial, se puede considerar como la creación o el mejoramiento de procesos, productos y servicios para ser comercializados, satisfaciendo los gustos y necesidades de los clientes, y obteniendo beneficios empresariales; esto conlleva al cambio a la transformación de las micro, pequeñas y medianas empresas (MIPYMES) con la finalidad de alcanzar un valor agregado para ser más competitivas y por lo tanto impactar a la sociedad. Por lo anterior, el objetivo principal de la investigación es determinar el rol de la innovación en empresas de Popayán, como factor de competitividad para lograr mejoramiento de la calidad de vida en la región.

La razón por la que se realiza la investigación en Popayán, es debido a que en la ciudad se encuentran un mercado que en su gran mayoría está compuesto por micro, pequeñas y medianas empresas. Según el informe de la Red ORMET (2013:58) el mercado de Popayán estaba compuesto en gran parte por microempresas en especial de artesanías, por tal motivo se debe reconocer la importancia de la innovación para que logren mayor sostenibilidad en el mercado, con el fin de adquirir organizaciones sostenibles y favorecedoras del mejoramiento de calidad de vida.

El Informe de la Cámara de Comercio del Cauca (2018) establece que, el $48 \%$ de las empresas se dedican al comercio al por mayor y al por menor de bienes en general, el $12 \%$ es el servicio de alojamiento y comidas y el $9 \%$ el sector manufacturero", así mismo, en la base de datos de la Cámara de Comercio del Cauca (2018), se encontraron registradas 25.916 empresas, de las cuales el $97 \%$ son micro, el $2.3 \%$ pequeñas, el $0.5 \%$ medianas y el $0.3 \%$ grandes empresas. Las MIPYMES se concentran en un $99.8 \%$, la investigación se centra en las MIPYMES comerciales y de servicios, identificando las empresas que presentan el componente innovador con 5 o más años de creadas, demostrando posicionamiento en el mercado de la ciudad, encontrándose en la curva de crecimiento, demostrando que si son exitosas.

Las empresas agregan valor $y$ producen riqueza para la economía del país, pero en el departamento del Cauca, en especial su capital Popayán, la mayoría de ella son micro y pequeñas que, según el informe de las 200 Empresas Generadoras de Desarrollo en el Cauca, realizado por la Cámara de Comercio del Cauca (2016:3), las microempresas conforman gran parte del "tejido empresarial, logrando crecimiento en un ambiente competitivo que les impulse a tener una visión más amplia de los mercados y a su vez les permita aprovechar los recursos y generar estrategias, favoreciendo así el ámbito de la innovación".

Es significativo que las empresas utilicen los recursos de la región para obtener mayor efectividad en los productos o servicios que necesita el mercado, con la finalidad de generar valor agregado para ser diferentes ante la competencia, además, las MIPYMES deben considerar mejoras, cambios y más conocimiento para ellas, esto apoyado en Sepúlveda \& Gutiérrez (2016:39), quienes piensan 
que "el tamaño afecta la supervivencia de las empresas, en este sentido, el ideal del tamaño para una empresa naciente sería aquel que pueda cubrir la demanda actual y que permita realizar inversiones escalonadas para cubrir la demanda futura". Popayán, cuenta con un desarrollo empresarial dedicado especialmente al comercio y a los servicios, en su mayoría conformado por MIPYMES, y se debe resaltar los aspectos innovadores empresariales que permiten a las empresas su crecimiento, obteniendo mayor valor agregado e impactando de manera directa en el aumento de la competitividad. Aspectos como implementar el uso de tecnologías, la innovación en procesos productivos, su ubicación geográfica, baja financiación, etc., son factores definitivos para la supervivencia empresarial.

En la investigación, se utilizó la metodología cualitativa de tipo exploratorio para detectar las variables, relaciones y condiciones de la innovación en las MIPYMES, se aplicó entrevista semiestructurada a treinta (30) MIPYMES de la ciudad de Popayán que permitió la recolección de información primaria. Para obtener los resultados se realizó un análisis discriminado entre las micro, pequeñas y medianas empresas, que posteriormente se unen para obtener resultados consolidados como los aspectos innovadores aplicados por ellas, entre los cuales se tienen que la innovación empresarial se cimentó en la implementación de lineamientos o tecnologías para mejoramiento continuo en procesos, productos y servicios.

\section{Innovación y competitividad}

Acorde con Leal \& Cefalá (2003:608), "las organizaciones se desenvuelven actualmente en un medio variable; caracterizado por avances tecnológicos, transformaciones políticas, sociales y económicas, que producen modificaciones en sus estructuras organizativas y exigen la reformulación de sus estrategias gerenciales, como manera de responder a las exigencias de un entorno cada vez más competitivo, incierto y globalizado", esto denota que el mercado es muy cambiante y cada vez más exigente, razón por la cual, la innovación es más importante, puesto que fomenta el crecimiento $y$ fortalecimiento; en muchas situaciones, el éxito empresarial es resultado de la aplicación de una cultura innovadora que ofrece soluciones, propuestas oportunas y eficientes para satisfacer al cliente.

Es relevante, tener en cuenta que la innovación genera valor y ofrece soluciones que satisfacen las necesidades y expectativas de los clientes, y no solo se trata de llevar al mercado un nuevo producto o servicio (Briceño, 2009). En este aspecto, es necesario entender que las empresas grandes no son las únicas que pueden ser innovadoras, puesto que éstas se pueden desarrollar en diferentes ámbitos, no hay que excluir a las micro, pequeñas y medianas empresas (MIPYMES), que debido a su flexibilidad pueden aprovechar la innovación mejorando su productividad y organización.

Se debe considerar la innovación como factor fundamental para las MIPYMES, que de acuerdo con González et al, (2014:110), se puede aplicar en "diferentes tamaños, rubros", espacios, principalmente en las empresas que quieren lograr el éxito empresarial; la innovación se orienta a la transformación de valor para obtener un mejoramiento, efectuando cambios en la forma de hacer las cosas, ensayando nuevas opciones para optimizar 
aspectos como: lo económico, funcional, estético, operacional, entre otros, o creando procesos nuevos, o utilizados en otros campos.

Por esto, es importante mencionar que la innovación utiliza recursos para llegar más allá encontrando oportunidades nuevas, analizando y desarrollando su aplicación, también se emplea para un producto, un servicio o a un proceso, realizando cambios en tecnologías, en gestión que signifiquen una innovación para la empresa o mercado, "la innovación es más que pura y simple capacidad de generar ideas nuevas" (Sainz, 2006:33).

Lo anterior, se complementa con el pensamiento de Mesa (2015:29), quien menciona que la innovación empresarial corresponde a un nuevo valor y no específicamente a algo nuevo, pero es importante considerar a Bernal \& Frost (2015:253) quienes analizan que, "para responder a las exigencias del nuevo ambiente de las organizaciones, en el campo de la innovación, ha surgido el enfoque de la innovación abierta que hace explícita la necesidad de apertura por parte de las empresas en sus procesos de innovación y pone énfasis en procesos como la práctica de rutinas de búsqueda de conocimiento externo como una de las principales fuentes para contribuir a potenciar la capacidad competitiva".

Para las empresas, es importante desarrollar habilidades innovar, que incluyan el diseño de servicios y desarrollo de modelos innovadores, programas de innovación, talleres de creatividad y nuevas ideas, acompañamiento empresarial, formación y entrenamiento de personal. Por esto, se puede indicar que las empresas innovadoras buscan cambios, evolucionan, hacen cosas diferentes, ofrecen nuevos productos y asumen procesos nuevos de producción. Hoy, deben ser innovadoras si buscan subsistir y diferenciarse de la competencia para ser más competitivas, ser conscientes que los productos y los procesos tienen un ciclo de vida cada vez más corto.

La empresa innovadora tiene equipos de trabajo comprometidos, con voluntad empresarial para asumir riesgos y sobre todo trabajo diario resolviendo problemas, satisfaciendo necesidades, siendo creativos, mejorando productos y servicios, sin miedo al cambio. Todo con el fin de ser líder en el mercado y sorprender a la competencia e incursionar en una "nueva economía basada en unos mercados cada vez más globalizados, apoyados en el vertiginoso desarrollo de la innovación tecnológica, sobre todo en lo relativo a sistemas de información" (Blázquez et al, 2006:47).

Es importante que las MIPYMES reconozcan y apliquen factores empresariales innovadores, deben ser propuestos y diseñados por el nivel gerencial para dar diferenciación a la empresa y contribuir a la obtención de ventajas competitivas cimentadas en componentes organizacionales como "procesos, productos, calidad, mercado, individuos sujetos a permanente actualización y cambio, como resultado de la aplicación de procesos de innovación" (Mathinson et al, 2007:66). Es indispensable tener un ciclo continuo de innovación, de esta manera permanecerán en el mercado, por lo cual, innovar es una necesidad para crear nuevos productos y servicios que tengan un valor adicional referente al tradicional trayendo consigo la preferencia y fidelidad de los clientes, de esta manera, se convierte en un factor de competitividad para las MIPYMES.

Blázquez, et al, (2006), se 
refieren a los factores empresariales internos, que condicionan o promueven el crecimiento, convirtiéndose en elementos que desde el contexto interno pueden generar potencialidades que fortalezcan el posicionamiento o generen ventajas competitivas, que algunas empresas han alcanzado promoviendo el crecimiento, la motivación, y la gestión del conocimiento. Respecto a los factores externos, se enfocan en la demanda, las mejoras tecnológicas, la accesibilidad a créditos privados y el apoyo gubernamental, los cuales son determinantes decisivos de las oportunidades y amenazas a las que se enfrentará en el futuro.

De otra parte, Suárez et al, (2007:381), concluyen que los factores innovadores del éxito empresarial de mayor incidencia en la innovación son: "una alta dirección abierta a nuevas ideas y proclive a asumir riesgos, la descentralización en la toma de decisiones y una dirección participativa, motivación y compromiso por la innovación en los directivos, formación constante del personal y ejecución de trabajo en equipo"; mientras que los factores externos fueron la "dinámica y turbulencia del entorno, que exige cambios, el establecimiento de vínculos y redes de cooperación en el país, la existencia de políticas estatales de apoyo a la innovación", facilidad de acceso a fuentes internacionales y nacionales de financiamiento para el desarrollo de la innovación, que es significativo para las MIPYMES, permitiéndoles obtener un mayor valor frente a sus competidores, les ofrece mejoramiento y logran sostenerse en el mercado aprovechando nuevas oportunidades que se presentan en el entorno.

Otro aspecto importante en la investigación es la competitividad, en este sentido, Solleiro \& Castañón (2005), presumen que la competitividad puede tener diferentes percepciones, se analiza desde diversos enfoques y disciplinas; por esta razón no se puede obtener una sola definición, esto depende de la necesidad de aplicación en las empresas para coadyuvar a incrementar niveles de mejoramiento. Sin embargo, diversos estudios sobre los factores que afectan la competitividad establecen que "la innovación actúa como común denominador, lo que implica que es una actividad esencial para todo tipo de empresas, sin tener en cuenta al sector, el ámbito de actuación, el tamaño y el enfoque", que se analizan para la competitividad; está aceptación, se da a partir de entender que la innovación es el elemento clave (Ibarra et al, 2017:382).

La competitividad es otro concepto importante y es visto como un factor primordial dentro de los mercados; proporciona expansión y prosperidad, se puede "llegar a la competitividad mediante cualidades superiores, unidas a las de cada industria, de la estructura del mercado y de los condicionantes generales del entorno en que se desenvuelven las empresas" (Gutiérrez et al, 2014:585).

El concepto de competitividad, toma importancia en el momento de unir a las empresas, en especial al identificar que las micro, pequeñas y medianas, representan un gran número a nivel mundial; por esto, se deben establecer las estrategias que las lleven a ser competitivas; el mercado es cada vez más exigente y las MIPYMES buscan crecimiento, estando inmersas en diversas áreas del mercado (Velásquez, 2004). Para que las MIPYMES logren el crecimiento deseado y sean más competitivas, deben organizar las rutinas establecidas, 
ser formales y eficientes con sus estructuras y procesos determinados, en entornos difíciles se debe analizar a la competencia con mayor innovación y tomar las ideas que son mejores para ellos (Bravo et al, 2015). Se debe tener aliados y establecer sinergias entre las organizaciones como estrategias en los procesos de desarrollo, de tal forma que permita el crecimiento de las empresas tanto a nivel local como regional.

Mora et al (2015:80), le dan importancia al desarrollo y fortalecimiento de estrategias de planeación para las MIPYMES en Colombia, "que les permitan proyectarse a mediano y largo plazo, y generar acciones que incrementen la competitividad frente al mercado internacional y que además les admitan no solo conservar el mercado que poseen actualmente, sino aumentar su radio de acción en nuevos mercados nacionales e internacionales".

Acorde a esto, se puede concluir que las MIPYMES deben identificar "factores claves de éxito, aprovechar la flexibilidad para adaptarse al entorno, potenciar los procesos internos de planificación, mejorar la gestión directiva, dirigiéndose hacia una cultura corporativa orientada a una mayor colaboración y participación de todos los miembros de la empresa"; obtener competencias que los diferencien, utilizando preferiblemente los recursos y capacidades intangibles (Saavedra et al, 2017:552).

Deben ser competitivas a partir de la innovación, las empresas pueden alcanzar un mejor posicionamiento $y$ crecimiento en el mercado, se debe considerar que están en crecimiento y un factor importante es que pueden establecer alianzas para fortalecer aspectos que pueden estar débiles.

\section{Micro, Pequeñas y Medianas Empresas (MIYMES): Análisis y discusión de resultados}

A partir de la aplicación de la entrevista semiestructurada, se analiza de manera independiente las micro, pequeñas y medianas empresas, comerciales y de servicios. Se determinan algunas variables importantes relativas a la innovación, identificadas por los empresarios entrevistados (número de empleados, Innovación como ventaja competitiva, desarrollo y difusión de nuevos y mejores procesos, nuevos productos o servicios, factores empresariales innovadores, factores internos innovadores, factores eternos innovadores, éxito empresarial relacionado con la innovación y el desarrollo de tecnologías) (cuadro 1).

En las variables se puede determinar que, existen resultados muy similares, la innovación es una ventaja competitiva, ésta apropiada a partir de la aplicación de nuevas tecnologías, sin olvidar que se debe satisfacer al cliente y se reconoce que les permite diferenciarse de la competencia, las pequeñas y medianas empresas buscan un mayor posicionamiento en el mercado, las medianas incursionan en los servicios virtuales y la participación de la innovación en la toma de decisiones. En cuanto al mejoramiento de los procesos, el más evidente es la atención al cliente, seguido por facturación, comercialización, servicios; en los procesos se han implementado aplicativos tecnológicos, programas de contabilidad e inventarios que les proporcionan mayor eficiencia. Existe siempre la preocupación de obtener nuevos productos o servicios; las micro se han enfocado en nuevos sistemas de 
crédito, negociaciones con proveedores, nuevos productos y marcas, las pequeñas implementan ventas en línea, reservas por canales electrónicos y las medianas ofrecen servicios financieros por plataformas virtuales.

Respecto a los factores empresariales innovadores, se coincide en buscar el mejoramiento de los procesos y la implementación de la calidad, se diferencias las pequeñas en la implementación de convenios y buscan exclusividad de productos a comercializar y servicios a ofrecer. Los factores internos identificados por los empresarios son la dirección abierta a nuevas ideas, la dirección participativa, formación contante del talento humano, motivación de líderes y empleados. La micro y medianas empresas concuerdan en asumir riesgos para obtener el cambio en la organización. Como factores externos innovadores las MIPYMES concuerdan en la dinámica y turbulencia del entorno, que hace importante estar atentos a todos los cambios y nuevas tendencias del mercado y las mejoras tecnológicas que permiten optimizar y mejorar los procesos actuales. Por lo anterior, el éxito de la empresa se relaciona con la innovación y el desarrollo de tecnologías, puesto que éstas ya se están implementado generando mejoras significativas en los procesos actuales que les ha permitido cumplir con las metas, los objetivos y las exigencias de los clientes.

\section{Cuadro 1 \\ Cuadro comparativo de variables determinantes para la innova- ción de las MIPYMES del sector comercial y de servicios}

\begin{tabular}{|c|c|c|c|}
\hline $\begin{array}{c}\text { Variables } \\
\text { Número de empleados }\end{array}$ & $\begin{array}{l}\text { Microempresas } \\
\qquad \text { De } 2 \text { y } 7\end{array}$ & $\begin{array}{l}\text { Pequeñas empresas } \\
\text { De } 11 \text { a } \mathbf{3 0}\end{array}$ & $\begin{array}{l}\text { Medianas empresas } \\
\text { De } 52 \text { a } 180\end{array}$ \\
\hline $\begin{array}{l}\text { Innovación como una } \\
\text { ventaja competitiva }\end{array}$ & $\begin{array}{l}\text { La innovación es una estrategia } \\
\text { para lograr una ventaja compe- } \\
\text { titiva en el mercado por medio } \\
\text { del uso de nuevas tecnologías } \\
\text { que diferencien los productos y } \\
\text { servicios ofrecidos por su cali- } \\
\text { dad, diseño moderno, creando } \\
\text { variedad para todos los gustos } \\
\text { con el propósito de satisfacer las } \\
\text { necesidades y expectativas de } \\
\text { los clientes. }\end{array}$ & $\begin{array}{l}\text { La innovación para diferenciarse } \\
\text { de la competencia directa e indi- } \\
\text { recta, adaptarse a los cambios y } \\
\text { a las tendencias actuales para } \\
\text { no desaparecer, satisfacer al } \\
\text { cliente, mejorar la eficiencia de } \\
\text { procesos, actualizar productos, } \\
\text { cumplir las exigencias de los } \\
\text { clientes, implementar nuevas } \\
\text { tecnologías, mejorar el servicio y } \\
\text { posicionarse en la ciudad. }\end{array}$ & $\begin{array}{l}\text { La innovación se asume como } \\
\text { una ventaja sobre la competen- } \\
\text { cia con el fin de posicionarse } \\
\text { y diferenciarse en el mercado, } \\
\text { cumplir metas, crecer y captar } \\
\text { nuevos clientes satisfaciendo } \\
\text { sus requerimientos de forma } \\
\text { única con productos y servicios } \\
\text { actualizados, esto con ayuda } \\
\text { de la implementación de nue- } \\
\text { vas tecnologías, variación de } \\
\text { productos, servicios virtuales } \\
\text { y la participación de la innova- } \\
\text { ción en la toma de decisiones. }\end{array}$ \\
\hline
\end{tabular}




\section{Cont... Cuadro 1}

Desarrollo y difusión de nuevos y mejores procesos
Se modifican y mejoran los procesos de atención al cliente, control de mercancías, inventarios, facturación, bases de datos, reciclaje y el proceso de transporte y distribución de productos. Dichas mejoras se realizan como resultado del análisis de mercados, que identifica las necesidades y como se podrían mejorar. Además, se logran la implementación de aplicativos tecnológicos actualizados y de fácil manejo, capacitación de procesos y de atención al cliente.
Se ha mejorado en procesos de atención al cliente, manejo de inventarios, facturación, ventas, mercadeo, diseños, calidad, promoción, base de datos y tramites, esto lo han logrado por medio de capacitaciones al personal, implementación de programas de computación, creación de páginas web, división del trabajo por áreas especializadas, análisis del mercado, identificación y corrección de inconsistencias y acuerdos con proveedores.

Las empresas gestionan la mejora en los procesos vitales para la actividad comercial o de servicios que realizan, obteniendo procesos más eficientes comparados con la competencia evitando la pérdida de tiempo y dándole mayor comodidad al cliente; con capacitaciones a personal en atención al cliente investigaciones realizadas para optar por nuevas y mejores formas de comercialización y prestar el servicio, diseño e implementación de modelos de gestión basados en las mejores prácticas y estándares de mercado, definiendo indicadores de desempeño reales, que aportan información para la optimización y sistematización de los procesos, alineados con la trasformación digital.

Se introducen nuevos productos y servicios al mercado, como los sistemas de créditos, negociaciones con proveedores de otras marcas poco comunes, productos de marcas nacionales e internacionales, nuevos diseños de productos acorde a las necesidades de los clientes que van de la mano con los cambios del entorno.
Se introducen nuevos servicios como mantenimiento, ventas online, reservas por canales electrónicos. Nuevos productos como cambio de colecciones acorde a las temporadas y fechas especiales, han puesto a la venta líneas de artículos diferentes, y actualización constante.
Las empresas implementan nuevos productos y servicios acorde a su actividad económica y a los cambios del entorno, oferta de servicios financieros por medio de plataformas virtuales, nuevos productos, herramientas sofisticadas, internet, televisión por cable o análoga.
- Mejoramiento de procesos especialmente en talento humano

- Mejoramiento de procesos

- Implementación de la calidad en productos y servicios,

Factores empresariales innovadores
- Variedad de productos y servicios,

- Actualización en tendencias del mercado.
- Buscar convenios con otras empresas para trabajos conjuntos.

- Implementación de la calidad en productos y servicios,

- Buscar exclusividad en productos y servicios

- Promoción y venta de productos de marcas reconocidas.
- Mejoramiento de procesos con aprovechamiento de tecnología,

- Implementación de un sistema de calidad, variedad de productos y servicios

- Crear y trasmitir las mejores prácticas,

- Mejoramiento continuo.
- Dirección abierta a nuevas ideas,

- Dirección participativa,

- Asumir riesgos para lograr el cambio en la organización,

Factores internos innovadores
- Descentralizar la toma de decisiones,

- Motivación de líderes y empleados

- Formación constante del personal, y el trabajo en equipo que genera mayor $y$ mejor calidad de vida
- Dirección participativa

- Motivación de líderes y empleados,

- Compromiso por la innovación,

- Formación constante del personal, y el trabajo en equipo que genera mayor y mejor calidad de vida.
- Dirección abierta a nuevas ideas,

- Dirección participativa,

- Asumir riesgos para lograr e cambio en la organización,

- Motivación de líderes y empleados,

- Compromiso por la innovación,

- Formación constante de personal, y el trabajo en equipo que genera mayor y mejor calidad de vida. 


\section{Colombia}

Saldarriaga Salazar, María Eugenia; Guzmán González, María Fernanda;

Concha Cerón, Eduardo Andrés

\section{Cont... Cuadro 1}

\begin{tabular}{|c|c|c|c|}
\hline $\begin{array}{l}\text { Factores externos inno- } \\
\text { vadores }\end{array}$ & $\begin{array}{l}\text { Dinámica y } \\
\text { turbulencia del entorno, que } \\
\text { hace importante estar atentos } \\
\text { a todos los cambios y nuevas } \\
\text { tendencias del mercado } \\
\text { Mejoras } \\
\text { tecnológicas que permiten } \\
\text { optimizar y mejorar los procesos } \\
\text { actuales. }\end{array}$ & $\begin{array}{l}\text { - Dinámica y } \\
\text { turbulencia del entorno, que } \\
\text { hace importante estar atentos } \\
\text { a todos los cambios y nuevas } \\
\text { tendencias del mercado, } \\
\text { Mejoras } \\
\text { tecnológicas que permiten } \\
\text { optimizar y mejorar los procesos } \\
\text { actuales. }\end{array}$ & $\begin{array}{l}\text { - Dinámica y } \\
\text { turbulencia del entorno, que } \\
\text { hace importante estar atentos } \\
\text { a todos los cambios y nuevas } \\
\text { tendencias del mercado } \\
\text { t Mejoras } \\
\text { tecnológicas que permiten } \\
\text { nuevas formas de realizar los } \\
\text { procesos para maximizar la } \\
\text { eficiencia Minimizar costos } \\
\text { y el desarrollo de productos. }\end{array}$ \\
\hline $\begin{array}{l}\text { El éxito de la empresa } \\
\text { relacionado con la inno- } \\
\text { vación y el desarrollo de } \\
\text { tecnologías }\end{array}$ & $\begin{array}{l}\text { El éxito de las micro tiene rela- } \\
\text { ción directa con la innovación e } \\
\text { implementación de nuevas tec- } \\
\text { nologías que ha logrado mejoras } \\
\text { significativas en los procesos } \\
\text { actuales de las empresas, cum- } \\
\text { pliendo así con las metas, los } \\
\text { objetivos y las exigencias de los } \\
\text { clientes. }\end{array}$ & $\begin{array}{l}\text { El éxito de las pequeñas } \\
\text { empresas se relaciona con la } \\
\text { innovación, generado ventajas } \\
\text { como: buen servicio al cliente, } \\
\text { actualización de productos y } \\
\text { servicios, diferenciarse de la } \\
\text { competencia, contar con equipo } \\
\text { de trabajo comprometido, } \\
\text { adoptar nuevas tecnologías, } \\
\text { todo esto generado el } \\
\text { crecimiento. }\end{array}$ & $\begin{array}{l}\text { El éxito de las medianas } \\
\text { empresas se relaciona } \\
\text { con la innovación ya que } \\
\text { permite el logro de las metas } \\
\text { empresariales, gestionando } \\
\text { la calidad, motivación, } \\
\text { compromiso, crecimiento } \\
\text { y valor agregado, además } \\
\text { de aprovechar el desarrollo } \\
\text { de nuevas tecnologías para } \\
\text { estandarizar procesos, } \\
\text { ejecución de tareas, control de } \\
\text { actividades y así mismo evaluar } \\
\text { el grado de cumplimiento de los } \\
\text { objetivos. }\end{array}$ \\
\hline
\end{tabular}

Fuente: Elaboración propia (2018)

A partir del cuadro anterior, se puede establecer que la innovación no es algo novedoso y se puede entrever que existe una fuerte relación con el marco económico y el aprovechamiento de las oportunidades que se encuentran en el contexto. Así mismo, la innovación es resultante del cambio por la indagación y construcción de una nueva forma de administrar las empresas, aunque aún se tienen algunos aspectos de estructuras anteriores. Se siente el impacto de la globalización en la economía, en la sociedad, en lo político y lo cultural, asumiendo que la innovación responda a la sociedad globalizada, con cambios que requieren evoluciones continuas y pueden ser parte de la solución a los problemas que se presentan en las MIPYMES comerciales y de servicios de Popayán, en su afán de estar adelante de los cambios y exigencias del mercado.

Para las MIPYMES, ya no es suficiente tener una ventaja competitiva o simplemente no tenerlas, es preciso diseñar e implementar variables nuevas que les permitan sostenerse en el mercado, desarrollarse y crecer. Las MIPYMES que han logrado éxito competitivo han realizado su planeación estratégica, innovan en sus procesos, productos, servicios, se preocupan por tener una mejor gestión, además, que demuestran mayor nivel tecnológico; se implementan lineamientos o tecnologías para mejoramiento continuo en procesos, productos y servicios.

Se entiende que la innovación debe ser un proceso continuo que da importancia a la iniciativa, las nuevas ideas y cambiando las estructuras establecidas, llevándolos al desarrollo de pensamientos que permiten la aplicación de servicios o mercados con ventaja competitiva. Se evidencian componentes innovadores fraccionados en factores 
internos y factores externos, enfocándose en instaurar una nueva forma de pensar y gestionar, entendiendo que la tecnología que se aplica en los diferentes procesos, ofrece a las MIPYMES una ventaja competitiva perdurable generando una mayor y mejor calidad de vida para todos al promover la capacitación del personal, se incrementan y actualizan sus conocimientos logrando un mejor desempeño que redunda en mayor productividad en las empresas.

Por el anterior análisis, se puede determinar que el rol de la innovación en las MIPYMES de la ciudad de Popayán es decisivo; éstas persiguen generar un mayor valor que sea duradero y conseguir una mejor sociedad. Se requiere ser innovadores y sobre todo estar constantemente revisando los procesos, así se evitar la desactualización, para esto se desarrolla el compromiso por la innovación. Es importante anotar, que los procesos de calidad que se están implementando en las MIPYMES, permiten evaluar el rendimiento y la inversión de la innovación efectuada para estar acorde a las metas planeadas.

Para las micro y pequeñas empresas va a ser un poco más difícil realizar los cambios por los pocos recursos que se tienen, pero igualmente, hacen grandes esfuerzos para lograrlo apoyándose en los recursos con los que se cuentan. Se deben buscar que la innovación sea más abierta, aprovechando todas las ideas que se generan, los conocimientos de las personas y realizar alianzas que pueden apoyar el fortalecimiento de las mismas. Las medianas empresas tienen más recursos y mayor facilidad para obtenerlos, por lo cual los procesos de innovación en ellas son más significativos.

Otro aspecto importante en la investigación es la competitividad de las MIPYMES, y para su análisis en las entrevistas a los empresarios se consideraron variables como crecimiento empresarial, organización de procesos, análisis de la competencia, influencia del entorno, aliados estratégicos y la gestión directiva (cuadro 2), en el cual se observa que las empresas si consideran su crecimiento, logrado en mayor medida por las pequeñas y medianas que buscan captar nuevos mercados y ven en la innovación la oportunidad para crecer porque con ella se obtiene competitividad en los mercados. La organización de los procesos es fundamental porque de esta manera se optimiza la labor de los trabajadores. El análisis de la competencia debe ser constante para evitar pérdida de clientes, pero se resalta que es necesario un componente que los diferencie de la competencia.

Los empresarios consideran que son influenciados por el entorno, con los cambios de la moda, las nuevas tendencias y la tecnología que ha sido necesario implementarla en la medida de las posibilidades económicas de las empresas. Los aliados estratégicos son importantes para el fortalecimiento empresarial, pero las pequeñas empresas si están implementado convenios para realizar trabajos conjuntos. La gestión directiva logra la colaboración y participación de todos los trabajadores de la empresa. 


\section{Cuadro 2}

Competitividad de las MIPYMES del sector comercial y de servicios: Análisis comparativo de variables determinantes

\begin{tabular}{|c|c|c|}
\hline Variables & Microempresas & Pequeñas empresas \\
\hline $\begin{array}{l}\text { Crecimiento de } \\
\text { las empresas }\end{array}$ & $\begin{array}{l}\text { Las empresas si consideran su } \\
\text { crecimiento, pero aun necesi- } \\
\text { tan más fortalecimiento, pero la } \\
\text { innovación es el aspecto más } \\
\text { importante para lograrlo }\end{array}$ & $\begin{array}{l}\text { Se ha considerado crecimiento de } \\
\text { la empresa al punto de tener la } \\
\text { posibilidad de destinar recursos } \\
\text { para innovación, nuevos puntos de } \\
\text { ventas con posibilidad de acceder } \\
\text { a nuevos mercados, cumplir con } \\
\text { el establecimiento de estándares } \\
\text { y alcanzar el reconocimiento en el } \\
\text { mercado a nivel regional, departa- } \\
\text { mental y nacional. }\end{array}$ \\
\hline
\end{tabular}

$\begin{array}{ll} & \begin{array}{l}\text { Las empresas son muy peque- } \\ \text { ñas pero han logrado identificar } \\ \text { sus procesos para mejorar las } \\ \text { actividades que realizan y las } \\ \text { procesos }\end{array} \\ \begin{array}{l}\text { personas obtienen una mejor } \\ \text { calidad de vida dentro y fuera } \\ \text { de la empresa }\end{array}\end{array}$

La competencia se está constantemente analizando, en el mercado hay muchas empresas similares y si utilizan nuevos servicios o comercializan Análisis de la otros productos, los clientes se competencia van a buscarlos, por esta razón hay que ofrecer cosas diferentes a los clientes para que prefieran la empresa, por eso se ha innovado en la implementación de tecnologías

Se considera que la influencia del entorno es muy fuerte, los Influencia del en- cambios de moda y tendencias torno afecta mucho éstas empresas y deben estar continuamente actualizándose.
Se cuenta con pocos procesos pero la dirección busca optimizar el trabajo de todos y las personas obtienen una mejor calidad de vida dentro y fuera de la empresa
Las empresas se adaptan a los cambios y a las tendencias actuales para no desaparecer. Se tuvo la necesidad de implementar nuevas tecnologías
Medianas empresas

Las empresas consideran que, si han tenido crecimiento, porque muchas de ellas nacieron como micro o pequeñas empresas, convirtiéndose hoy en medianas empresas, y contemplan la innovación como factor importante para seguir creciendo y ser reconocidos en otros mercados, captar nuevos clientes satisfaciendo sus requerimientos de forma única.

Se gestiona la mejora de los procesos, buscando que sean más eficientes de esa manera se optimiza el tiempo y se ofrece mejor atención al cliente. Las personas obtienen una mejor calidad de vida dentro y fuera de la empresa

La competencia se debe vigilar de manera continua, para evitar ser inferiores a ellos, se debe tener un componente diferenciador, que logre la preferencia de los clientes y la innovación es manera de lograr la ventaja competitiva

Analizando la competencia se puede obtener unos procesos más eficientes y evitar pérdida de tiempo en la empresa. Hay que adquirir un componente diferenciador de la competencia para que los clientes prefieran la empresa e innovando en algunos aspectos de la empresa se ha logrado la diferenciación.

La influencia del entorno es fuerte y llevo a las empresas a la implementación de nuevas tecnologías, ventas en línea

Se han buscado convenios con otras empresas para realizar trabajos conjuntos.

Estas empresas consideran importantes los aliados estratégicos, pero no se evidencia el trabajo conjunto con otras empresas

La gestión del empresario logra la colaboración y participación de todos los trabajadores de la empresa

Fuente: Elaboración propia (2018) 
En los resultados de los cuadros, se puede evidenciar que los empresarios consideran que la innovación les ofrece la diferenciación que es necesaria para distinguirse de la competencia y los lleva a ser más competitivos en el mercado. Por esta razón, es importante estar constantemente analizando la competencia que ha implementado mayor innovación, y obtener las mejores ideas, además atender los cambios que el entorno genera. Para que las MIPYMES logren crecimiento y sean más competitivas, deben organizar sus procesos y la labor de los trabajadores para ser más eficientes en entornos difíciles. Las empresas reconocen que para ser competitivas deben ser innovadoras, de esta manera logran posicionamiento e incremento en el mercado y por consiguiente se obtiene una mejor calidad de vida en la región. Se busca diseñar estrategias para ser más competitivas, y conocen las exigencias del mercado, de esta manera, muchas de las pequeñas y medianas empresas han logrado crecimiento.

\section{Conclusiones}

En la investigación, se determina que la innovación es fundamental para alcanzar la competitividad empresarial. Las MIPYMES ya están avanzando en la implementación de cambios o mejoramientos en sus procesos que las convierte en innovadoras, llevándolos a la competitividad y les permitan diferenciarse de la competencia. Se puede concluir que acogen las propuestas de la gestión directiva, y por el tamaño de éstas empresas, pueden ser más flexibles y es más fácil realizar los cambios que, en las grandes empresas por el riesgo que deben asumir. Por esto, es preciso conocer cómo afecta la innovación a la competitividad de las MIPYMES.

Las empresas, buscan su mejoramiento y al obtenerlo se logra el mejoramiento de la calidad de vida de sus trabajadores y como consecuencia en la región, y lo hacen aplicando innovación de diferentes maneras acorde a la estructura de la empresa; algunas empresas implementan cambios en los procesos, o en productos a comercializar, o en los servicios, o en la mejora de la atención al cliente que ha sido un factor muy importante para las MIPYMES analizadas, como consecuencia de esto se ha logrado la competitividad, con la habilidad de proveer productos y servicios con mayor eficiencia y eficacia frente a la competencia, estando acorde a las nuevas tendencias, para implementar los cambios en cuanto a productos a comercializar y servicios.

este último se centra en estar en evolución acorde a las nuevas formas de realizar los procesos para maximizar la eficiencia, minimizar costos, ofrecer nuevos o renovados productos $y$ servicios, las empresas deben estar al tanto de las nuevas tendencias, para implementar las novedades en cuanto a productos a comercializar y servicios con el fin de competir y satisfacer sus segmentos de mercado.

La innovación para las MIPYMES de comercio y servicio de la ciudad de Popayán, es fundamental en aspectos como: el logro de las metas propuestas, la dirección abierta a nuevas ideas, la dirección participativa, la asunción de riesgos, la motivación de líderes y empleados, compromiso por la innovación, formación constante del personal, y el trabajo en equipo, 
y además, el desarrollo de nuevas tecnologías para estandarizar procesos, ejecución de tareas, control de actividades y así mismo evaluar el grado de cumplimiento de los objetivos. La innovación le ha permitido a las MIPYMES lograr la competitividad que las ha llevado al posicionamiento en el mercado, satisfaciendo las necesidades de sus clientes con el mejoramiento de los servicios, productos y procesos para que sean más competitivas. La innovación le admite a las MIPYMES tener una diferencia en el sector de servicios y comercio en el cual la competencia es muy fuerte en la ciudad, pero logran estar un paso adelante en el mercado local, considerando como la habilidad de desarrollar pensamientos que permitan la aplicación nuevas ideas de servicio o mercadeo con una ventaja competitiva.

Para las empresas estudiadas, es difícil destinar grandes cantidades de dinero y recursos para innovar; solo las empresas más grandes tienen la solvencia para realizarlo. Pero, se demostró que las MIPYMES pueden innovar utilizando menos recursos, con el mejoramiento de sus procesos, la implementación de sistemas de Software, hasta cambios tecnológicos, manejo de medios virtuales como páginas web o aplicaciones para celular, inversiones que están al alcance de las ellas.

Principalmente, el valor agregado que caracteriza a las MIPYMES es la asesoría y la buena atención a todos los clientes en cuanto a los productos o servicios que desean averiguar o adquirir, ofreciendo una atención personalizada y permitiendo conocer muy bien las características y ventajas de lo que se ofrece.

El tamaño, si hace diferencia en la necesidad de aplicar la innovación, pues las micro se centran en mejorar el método de facturación, manejo de bases de datos e inventarios con el fin de mejorar procesos, además de la búsqueda de una ventaja competitiva para ser diferentes en el mercado que los lleve a ser más competitivos, las pequeñas y medianas empresas, agregado a lo anterior también han dado pasos hacia el campo virtual, manejando páginas web para venta y promoción, por otra parte las medianas se enfocan en realizar inversiones a mayor escala como la creación de modelos de gestión, realización de investigaciones, uso de software específicos, introducción de nuevos productos, servicios, procesos y tecnologías, como el manejo de medios virtuales para dar productos y servicios de una forma eficiente y cómoda para el cliente.

A nivel externo los factores que influencian a las empresas hacia la innovación primordialmente son la dinámica y turbulencia del entorno y las mejoras tecnológicas, pero estos factores son positivos para las MIPYMES, porque les ha permitido estar constantemente mejorando, acoger los cambios acordes a sus capacidades y seleccionan los productos a comercializar y los servicios novedosos para sus clientes. Se ha implementado la tecnología en algunos de sus procesos, generando mayor eficiencia en la atención al cliente.

\section{Referencias Bibliográficas}

Bernal, César Augusto, y Frost, Salomón (2015), Innovación abierta en empresas colombianas: reto a superar. Revista Venezolana de Gerencia (RVG), Vol. 20, No 70, Maracaibo, Venezuela. Pp.252-267.

Blázquez, Félix; Dorta, José Andrés y Ve- 
rona, María Concepción (2006), Factores del crecimiento empresarial. Especial referencia a las pequeñas y medianas empresas. INNOVAR, Revista de Ciencias Administrativas y Sociales, Vol 16, No 28. Bogotá, Colombia. Pp. 43-56.

Bravo, Saulo; Azcárate, Jacinto; Rojas, Alexander y Narváez, Carlos (2015), Análisis Comparativo de Estrategias y Competitividad entre Mipymes. Desarrollo Gerencial, Vol 1, No 7. Barranquilla, Colombia. Pp. 102-121.

Briceño, María de los Ángeles (2009), La innovación como accionante del Emprendimineto en las Organizaciones. Revista EAN, No 66. Medellín Colombia. Pp. 85-98.

Cámara de Comercio del Cauca (2016), 200 Empresas Generadoras de Desarrollo en el Cauca. Popayán, Editorial EI Nuevo Liberal.

Cámara de Comercio del cauca (2018), Análisis de la Supervivencia de las empresas den Popayán y el Cauca.Popayán, El Nuevo Libera.

Flick, Uwe. (2015), El Diseño de Investigación Cualitativa. Madrid, España. Ediciones Morata, S. L. Pp.168

González, Julio; García, Luis; Caro, Carla Lucero y Romero, Natalia. (2014), Estrategia y cultura de innovación, gestión de los recursos y generación de ideas: prácticas paragestionar la innovación en empresas. Pensamiento \& Gestión, No 36. Barranquilla, Colombia. Pp. 109-135.

Gutiérrez Verónica; Medina, José Francisco; Viesca, Felipe Carlos y Favil, Héctor (2014), La Competitividad de la Micro y Pequeña Empresa de Alimentos y Bebidas. El casodel municipio turístico de Metepec en el Estado de México. Estudios y Perspectivas en Turismo, Vol 23, No 3. Buenos Aires, Argentina. Pp. 585-606.
Ibarra, Manuel Alejandro; González, Lourdes Alicia y Demuner, Maria del Rosario. (2017), Competitividad empresarial de las pequeñas y medianas empresas manufactureras de Baja California. Estudios Fronterizos, Vol 18, No 35. Baja California, Mexico. Pp.107-130.. Doi:10.21670/ ref.2017.35.a06

Kerlinger, Fred y Lee, Howard (2005), Investigación del Comportamiento. Métodos de Investigación en Ciencias Sociales. México, Editorial Mc Graw Hill. Pp 936

Leal, Miraidy; Cefalá, Yilian (2003), Estrategias de crecimiento empresarialaplicadas por hipermercados. Revista Venezolana de Gerencia (RVG), Vol 8, N 24. Maracaibo, Venezuela. Pp. 607-621. Recuperado el 25 de Noviembre de 2018, de http:// www.redalyc.org/pdf/290/29002406. pdf

Mathinson, Luis; Gándara, José; Primera, Carlos y Garcia, Luis (2007). Innovación: Factor Clave para Lograr Ventajas Competitivas. Revista NEGOTIUM, Vol 3 No 7. Pp.46-83.

Mesa, Jorge Hernán (2015). Emprendimientos Innovadores latinoamericanos. Medellin, Colombia, Fondo Editorial Universidad EAFIT. Pp.326

Mora, Edwin H; Vera, Mary A y Melgarejo, Zuray A. (2015), Planificación estratégica y niveles de competitividad de las Mipymes del sector comercio en Bogotá. Estudios Gerenciales, Vol 31 Cali, Colombia. P.p 79-87. Doi:https://doi.org/10.1016/j.estger.2014.08.001

RED ORMET, Red de Observatorios del Mercado de Trabajo (2013), Informe de Diagnóstico, Mercado de Trabajo Popayán. Departamento de Prosperidad Social - DPS. Bogotá, Colombia. Programa de Naciones Unidas para el Desarrollo. 
Innovación Empresarial: Factor de competitividad y calidad de vida en Popayán,

Colombia

Saldarriaga Salazar, María Eugenia; Guzmán González, María Fernanda;

Concha Cerón, Eduardo Andrés

Saavedra, María Luisa; Camarena, María Elena yTapia, Blanca (2017), Calidad para la competitividad en las micro, pequeñas y medianas empresas, de la Ciudad de México. Revista Venezolana de Gerencia (RVG), Vol 22, No 80. Maracaibo, Venezuela. Pp. 551-575. Recuperado el 18 de Febrero de 2019, de

Sainz de Vicuña, José María. (2006). Innovar con éxito. Madrid, España. ESIC Editorial. Pp.295

Sandoval, Carlos A. (2002). Investigación Cualitativa. Bogotá, Colombia: ARFO Editores e Impresores Ltda. Pp. 313.

Sepulveda, Claudia Ines y Gutiérrez, Reina (2016). Sostenibilidad de los emprendimientos: Un análisis de los factores determinantes. Revista Ve- nezolana de Gerencia (RVG), Vol 21, No 73. Maracaibo, Venezuela. Pp. 33-49..

Solleiro, José Luis y Castañón, Rosario. (2005), Competitiveness and innovation systems: the challenges for México's insertion in the global contex. Technovation. Vol 25 No 9, 10591070.

Suárez, Jesús.; Albisu, L.M.; Sotolongo, Naicy y Blanco, F. (2007), Factores de éxito y fracaso en el comportamiento innovador. Pastos y Forrajes, Vol 30, No 3. Matanzas, Cuba. Pp. 381-394.

Velásquez, Francisco (2004), La estrategia, la estructuta y las formas de asociación: fuentes de ventaja competitiva para las pymes colombianas. Estudios Gerenciales, No 93. Cali, Colombia. Pp 73-98.

- Esta obra está bajo una licencia de Creative Commons Reconocimiento-NoComercialCompartirlgual 3.0 Unported. http://creativecommons.org/licenses/by-nc-sa/3.0/deed.es_ES 